\section{OROPHARYNGEAL SUCTIONING IN NEWBORN INFANTS IMPAIRS CEREBRAL OXYGENATION DURING TRANSITION AFTER BIRTH}

\author{
G. Pichler, M. Pocivalnik, W. Raith, H. Zotter, \\ E. Ziehenberger, W. Müller, B. Urlesberger
}

Pediatrics, Medical University Graz, Graz, Austria

Background: Oropharyngeal suctioning during transition after birth can induce bradycardia and/ or apnoea. These are reasons for restrictive approach.

Objectives: Aim of the study was to investigate the influence of oropharyngeal suctioning on cerebral and peripheral oxygen saturation during transition after birth.

Methods: In a prospective randomized study term newborn infants were measured during the first ten minutes after elective caesarean section. In the suctioning group oropharyngeal suctioning was done for five seconds in the first minute of life. In the control group no suctioning was done.

Regional tissue oxygenation (rSO2) was measured of the brain, preductal peripheral tissue (right forearm) and postductal (left calf) peripheral tissue using near-infrared-spectroscopy (INVOS 5100, Somanetics). Heart rate and arterial oxygen saturation $(\mathrm{SaO} 2)$ were measured preductal (right hand) and postductal (left foot) with pulseoxymetry.

Results: 20 newborn infants were included in each group. All showed normal adaptation.

Five minutes after birth in the suctioning group cerebral rSO2 $(58+/-23 \%$ vs. $70+/-13 \%)$ and postductal $\mathrm{SaO} 2(70+/-19 \%$ vs. $82+/-11 \%)$ were significantly lower compared to the control group. rSo2 of preductal $(44+/-22 \%$ vs. $51+/-17 \%)$ and postductal peripheral tissue $(36+/-19 \%$ vs. $39+/-$ $13 \%)$, as well as preductal $\mathrm{SaO} 2(80+/-13 \%$ vs $84+/-8 \%$ ) tended to be lower in the suctioning group compared to the control group, but without reaching significance. Ten minutes after birth, there were no significant differences.

Heart rate did not show significant differences between both groups.
Conclusion: This study demonstrated that oropharyngeal suctioning impairs cerebral tissue oxygenation (rSO2) and postductal $\mathrm{SaO} 2$ during transition after birth.

\section{3}

\section{THE ROLE OF ENDOTHELIAL PROGENITOR CELLS IN NEOVASCULARIZATION OF ISCHEMIC RETINA THROUGH SDF-1/CXCR4 AXIS IN OXYGEN-INDUCED RETINOPATHY RAT MODEL}

\author{
A. Villalvilla ${ }^{1}$, M. Moro' ${ }^{1}$, L. Arruza ${ }^{1}$, G. Villar ${ }^{1}, \mathrm{G}$ \\ Herranz ${ }^{1}$, A. Fernandez-Cruz ${ }^{2}$, \\ R. Fernandez-Durango ${ }^{3}$ \\ ${ }^{1}$ Neonatology, ${ }^{2}$ Internal Medicine III, ${ }^{3}$ Research \\ Unit. Internal Medicine III., Hospital Clinico San \\ Carlos, Madrid, Spain
}

Retinopathy of prematurity (ROP) is a common cause of blindness in preterm infants worldwide, despite laser treatment. Animal models of oxygeninduced retinopathy (OIR) have been developed to investigate physiological events that lead to the occurrence of this disease. It is known that endothelial progenitor cells (EPCs), derived from bone-marrow, are involved in vascular repair and neovascularization. In the present study we investigate the retinal expression of the SDF-1/ CXCR4 axis, its role in the recruitment of EPCs to the hypoxic retina, and the relationship between these cells and the development of neovascularization in a 50/10 OIR rat model. Our results show increased production of SDF-1 by endothelial cells and neurons in OIR retinas, and enhanced expression of CXCR4 mainly in Müller cells. We have also found in peripheral blood of OIR rats a decreased number of colony-forming units of EPCs. Whether this is due to the presence of functionally impaired EPCs or to the inability of an exhausted bone-marrow to produce enough progenitor cells to cope with the high demands of the process of retinal neovacularization, remains unanswered. We demonstrate in this study that SDF-1/CXCR4 axis, EPCs dysfunction and probably Müller cells, are involved in retinal neovascularization in a 50/10 rat model of OIR. These results warrant further studies. 